\title{
Ultrasound Assessment of Lung Aeration in Subjects Supported by Venovenous Extracorporeal Membrane Oxygenation
}

\author{
Xiao Lu, Charlotte Arbelot, Annia Schreiber, Olivier Langeron, Antoine Monsel, and Qin Lu
}

\begin{abstract}
BACKGROUND: The value of ultrasound in assessing lung aeration of patients with ARDS who require venovenous extracorporeal membrane oxygenation (ECMO) has, to our knowledge, never been studied. The objective of the study was to evaluate by using ultrasound lung aeration at ECMO initiation and withdrawal in subjects with severe ARDS supported by venovenous ECMO. METHODS: Fifty subjects were included in this pilot retrospective study. The lung ultrasound aeration score (LUS) and respiratory variables were collected at ECMO initiation (TO) and ECMO withdrawal (T1). The LUS at T0 between the subjects who survived to ICU discharge and those who died in ICU was compared. The relationship between changes in LUS and changes in $\mathrm{P}_{\mathrm{aO}_{2}} / \mathrm{F}_{\mathrm{IO}_{2}}$ from T0 to T1 was assessed. RESULTS: The ICU mortality was $34 \%$. The LUS at T0 did not differ between survivors and non-survivors (median 22 [interquartile range] $\{$ IQR $\}$ 19-26 vs median 24 [IQR, 19-28]; $P=.60$ ). From T0 to T1, the LUS decreased significantly in survivors (median 22 [IQR, 19-26] vs median 16 [IQR, 13-19]; $P<.001)$, it decreased moderately in non-survivors who were weaned off ECMO (median 26 [24-29]) vs median 22 (IQR, 17-24), $P=.031$ ), and remained stable in those who died during ECMO (median 25 [IQR, 19-29] vs median 25 [IQR, 23-31]; $P=.22$ ). Changes in $\mathrm{P}_{\mathrm{aO}} / \mathrm{F}_{\mathrm{IO}_{2}}$ were not related to changes in the LUS between T0 and T1. CONCLUSIONS: At the time of ECMO placement, the subjects who survived ARDS had aeration loss close to that observed in the subjects who did not survive. At the time of ECMO withdrawal, there was a significant improvement in lung aeration in the survivors, whereas a severe loss of lung aeration persisted in the non-survivors, although some were weaned off ECMO. Lung ultrasound provided a valuable tool for bedside assessment of lung aeration in subjects supported by ECMO. Key words: ARDS; ultrasound; VV ECMO; lung aeration; oxygenation; survivors; non-survivors. [Respir Care 2019;64(12):1478-1487. (C) 2019 Daedalus Enterprises]
\end{abstract}

\section{Introduction}

Venovenous extracorporeal membrane oxygenation (VV ECMO) is a treatment option for patients with ARDS re-

Drs X Lu, Arbelot, Schreiber, Langeron, Monsel, and Q Lu are affiliated with the Multidisciplinary Intensive Care Unit, Department of Anesthesiology and Critical Care, La Pitié-Salpêtrière Hospital, Assistance PubliqueHôpitaux de Paris, Sorbonne Université, Paris, France. Dr X Lu is affiliated with the Department of Emergency Medicine, Second Affiliated Hospital, Zhejiang University School of Medicine, Hangzhou, China. Dr Schreiber is affiliated with the Division of Respirology, Department of Medicine, University Health Network, Toronto, Ontario, Canada. Dr Langeron is affiliated with the Department of Anesthesiology and Critical Care, Henri-Mondor Hospital, Assistance Publique-Hôpitaux de Paris, Créteil, France.

The authors have disclosed no conflicts of interest. fractory to conventional therapies. ${ }^{1,2}$ Despite the increasing use of VV ECMO, in-hospital or 60-d patient mortality remains high. ${ }^{1,3,4}$ Many factors have been demonstrated as being independently associated with mortality during ECMO: age, 5 duration of mechanical ventilation before ECMO, pulmonary compliance, driving pressure, and ARDS associated with extrapulmonary organ dysfunction. ${ }^{6-8}$ In ARDS, it has been reported that the initial degree of lung aeration loss as well as the changes in lung

\footnotetext{
Correspondence: Qin Lu MD PhD, Réanimation Chirurgicale Polyvalente, Département d'Anesthésie-Réanimation, Hôpital Pitié-Salpêtrière, 47-83 Boulevard de 1'Hôpital, 75013 Paris, France. E-mail: qin.lu@aphp.fr.
}

DOI: $10.4187 /$ respcare. 06907 
aeration after applying a new ventilatory strategy could affect the mortality of patients with ARDS. ${ }^{9,10}$ In patients with severe ARDS and who require ECMO support, it is still unknown whether the severity of lung aeration loss at the onset of ECMO could influence the outcome in these patients. In addition, among the criteria commonly used for ECMO weaning, the recovery of lung function attested by pulmonary re-aeration is not necessarily considered.

Lung transthoracic ultrasound is increasingly used in the ICU for bedside assessment of PEEP-induced alveolar recruitment, ${ }^{11}$ antibiotic-induced lung re-aeration in ventilator-associated pneumonia, ${ }^{12}$ and lung aeration changes during a spontaneous breathing trial. ${ }^{13}$ Lung aeration assessed by ultrasound is correlated with lung volume assessed by computed tomography. ${ }^{11,12}$ To date, lung aeration changes in patients with ARDS who require VV ECMO have only been reported in a few patients. ${ }^{14}$ Thus, the primary objective of the present study was to evaluate lung aeration by ultrasound at the beginning of the ECMO in the subjects who survived to ICU discharge and those who died in ICU. Secondary objectives were changes in lung aeration from ECMO initiation to ECMO withdrawal and the relationship between lung re-aeration and improvement of oxygenation in subjects who received VV ECMO.

\section{Methods}

\section{Subjects and Study Protocol}

This was a pilot retrospective cohort study. Subjects were recruited between April 2012 and March 2018 from 2 multidisciplinary ICUs of a university hospital. Inclusion criteria were patients with severe ARDS ${ }^{15}$ supported by VV ECMO assistance. The indication for the establishment of VV ECMO was $\mathrm{P}_{\mathrm{aO}} / \mathrm{F}_{\mathrm{IO}_{2}}<$ of $60 \mathrm{~mm} \mathrm{Hg}$ for $\geq 3 \mathrm{~h}$ or of $<90 \mathrm{~mm} \mathrm{Hg}$ for $>6 \mathrm{~h}$, and/or refractory respiratory acidosis $(\mathrm{pH}<7.25)$, with inspiratory plateau pressure of $\geq 32 \mathrm{~cm} \mathrm{H}_{2} \mathrm{O}$ despite optimization of conventional therapy. In our ICU, transthoracic lung ultrasound is routinely performed to monitor changes in lung aeration in patients with ARDS.

Each subject was assessed by transthoracic lung ultrasound from -24 to $24 \mathrm{~h}$ after the initiation of ECMO (T0), and from -24 to $24 \mathrm{~h}$ of ECMO withdrawal, or the last lung ultrasound assessment before ECMO removal if the subject died during ECMO support (T1). Exclusion criteria were patients without lung ultrasound assessment between $-24 \mathrm{~h}$ and $24 \mathrm{~h}$ after the onset of ECMO or patients in whom assessment by lung ultrasound did not include total lung regions due to the presence of subcutaneous emphysema or pleural drainages.

According to the French law on ethics on retrospective studies, written informed consent of subjects or their relatives was waived, but the information that concerned the study was explained to the subjects and consent to data collection was

\section{QUICK LOOK}

\section{Current knowledge}

The initial degree of lung aeration loss could affect the mortality of patients with ARDS. In patients who receive venovenous extracorporeal membrane oxygenation (ECMO), it is unknown whether the severity of lung aeration loss at onset and withdrawal of ECMO influences the outcome.

\section{What this paper contributes to our knowledge}

At the time of ECMO placement, the subjects who survived ARDS had lung aeration loss close to that observed in the subjects who did not survive. At the time of ECMO withdrawal, a significant improvement in lung aeration was obtained in the survivors, whereas a severe loss of lung aeration persisted in non-survivors, although some were weaned off ECMO. Lung ultrasound provides a valuable tool for bedside assessment of lung aeration in subjects supported by ECMO.

requested from the subjects. The study was entered into the register of data protection of the study institution. The study was performed in the Multidisciplinary Intensive Care Unit, Department of Anesthesiology and Critical Care, La PitiéSalpêtrière Hospital, Assistance Publique-Hôpitaux de Paris, Sorbonne Université, Paris, France.

\section{Lung Ultrasound Assessment}

Transthoracic lung ultrasound was performed either by an experienced physician with level- 3 certification ${ }^{16}$ or by a resident or a senior physician who had completed lung ultrasound training. ${ }^{17}$ A Siemens Acuson CV70 (Simens Medical Solutions, Malvern, PA) or a Philips Sparq (Philips Ultrasound, Bothell, WA) ultrasound device equipped with a 2- to $5-\mathrm{MHz}$ convex probe was used for the examination. In each subject, upper and lower lung areas of the right and left lungs were delineated by the parasternal, anterior axillary, and posterior axillary and paravertebral lines. Therefore, 12 lung regions that corresponded to antero-superior, antero-inferior, latero-superior, latero-inferior, postero-superior, and posterior-inferior lung areas were examined. ${ }^{13} \mathrm{~A}$ numeric value was assigned to each area according to the most-severe lung ultrasound finding detected in the corresponding intercostal space as follows (Fig. 1): 0, normal aeration (defined by the presence of lung sliding with horizontal A lines or fewer than 2 isolated vertical B lines); 1, moderate loss of lung aeration (defined as the presence of either multiple well-defined and spaced B1 lines issued from the pleural line or from small juxtapleural consolidations and correspond to interstitial 

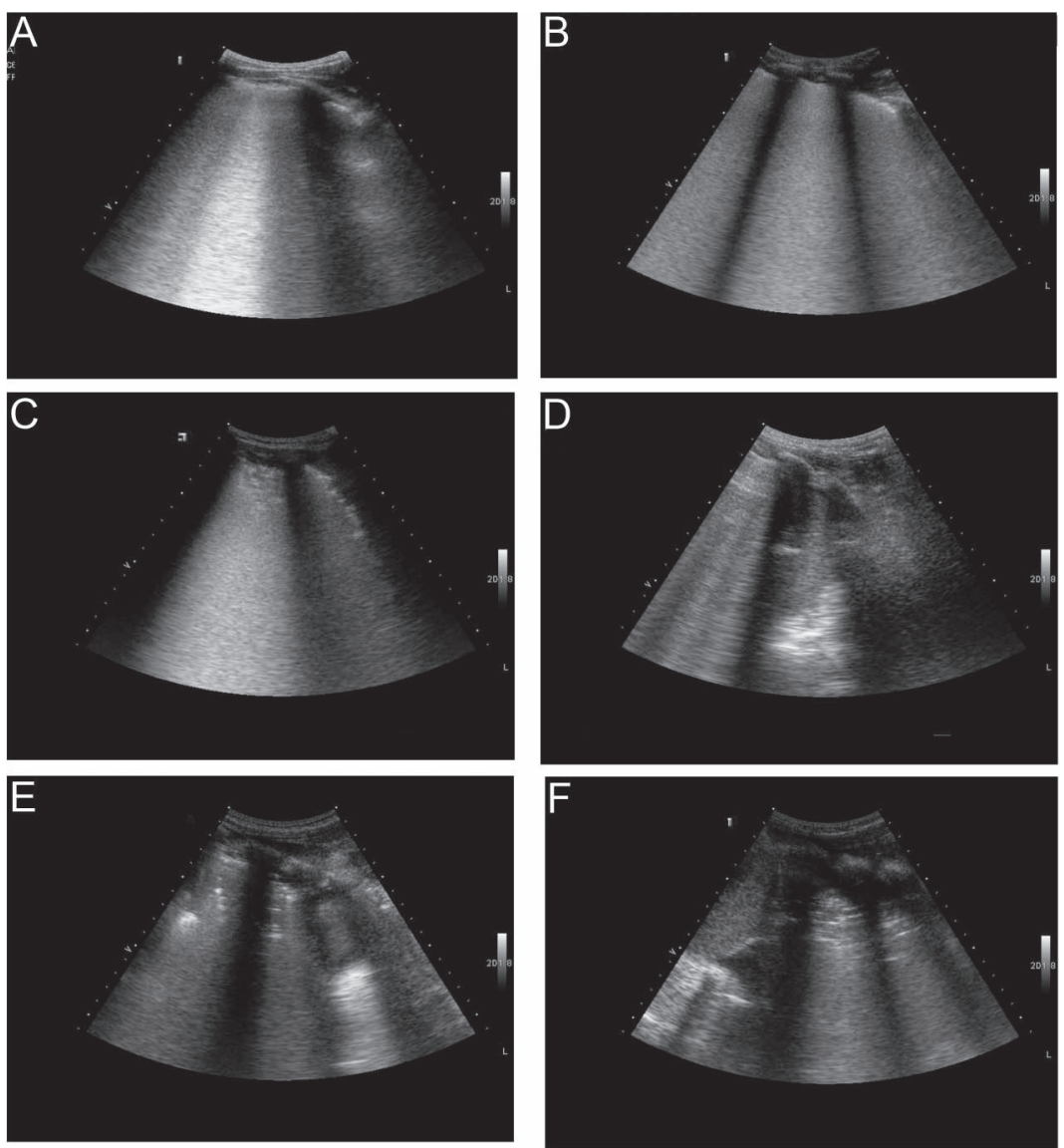

Fig. 1. Ultrasound assessment of lung aeration. A-F: Lung ultrasound images of 6 examined lung areas (antero-superior, antero-inferior, latero-superior, latero-inferior, postero-superior, and postero-inferior parts of the right lung) in a subject of the study. A numeric value was assigned to each area according to the most-severe lung ultrasound finding. A-C: Multiple coalescent vertical B2 lines issued from the pleural line. C-F: The presence of a tissue pattern that contains either hyperechoic punctiform or linear images.

edema, or coalescent B1 lines issued from the pleural line or from small juxtapleural consolidations, present in a limited portion of the intercostal space, which correspond to localized alveolar edema); 2 , severe loss of lung aeration (multiple coalescent vertical B2 lines issued either from the pleural line or from juxtapleural consolidations, detected in the whole area of one or several intercostal spaces, which correspond to diffuse alveolar edema); and 3, lung consolidation (defined as the presence of a tissue pattern that contains either hyperechoic punctiform or linear images representative of static air bronchograms, or the same images with inspiratory centrifugal movement, representative of dynamic air bronchograms, ${ }^{18}$ which correspond to complete loss of aeration). ${ }^{19}$ The lung ultrasound aeration score (LUS) was calculated as the sum of the numeric values assigned to each lung zone, ranging from 0 to $36 .{ }^{13,20}$

\section{VV ECMO Technique}

The cannulae were percutaneously inserted: a large venous drainage cannula was inserted into the femoral vein up to the right atrium, and the return cannula carried oxygenated blood into the jugular vein to the superior vena cava. If this was not possible, then the opposite femoral vein was cannulated to the lower part of the inferior vena cava. Cannula insertion was always accompanied by transesophageal or transthoracic echocardiography to ensure correct placement. The VV ECMO technique consisted of Raumedic cannulas (Raumedic, Helmbrechts, Germany), a PLS-i membrane oxygenator with BIOLINE coating (Maquet, Rastatt, Germany), and a Rotaflow RF32 centrifugal pump and console (Maquet). In some cases, a CARDIOHELP device (Marquet) was used. Anticoagulation was achieved with unfractionated heparin with anti-Xa activity between 0.2 and $0.3 \mathrm{IU}$ per $\mathrm{mL}$ unless contraindicated. ${ }^{1,21}$

An ECMO weaning test was performed by decreasing the membrane ventilation to $0 \mathrm{~L} / \mathrm{min}$ for at least $1 \mathrm{~h}$ when clinical improvements were observed. The ECMO device could be withdrawn if $\mathrm{P}_{\mathrm{aO}_{2}}$ was $>70 \mathrm{~mm} \mathrm{Hg}$, with $\mathrm{F}_{\mathrm{IO}_{2}}$ $<60 \%$, inspiratory plateau pressure $<30 \mathrm{~cm} \mathrm{H}_{2} \mathrm{O}$ with tidal volume $<6 \mathrm{~mL} / \mathrm{kg}$, and if echocardiography did not reveal evidence of acute cor pulmonale. ${ }^{1}$ 


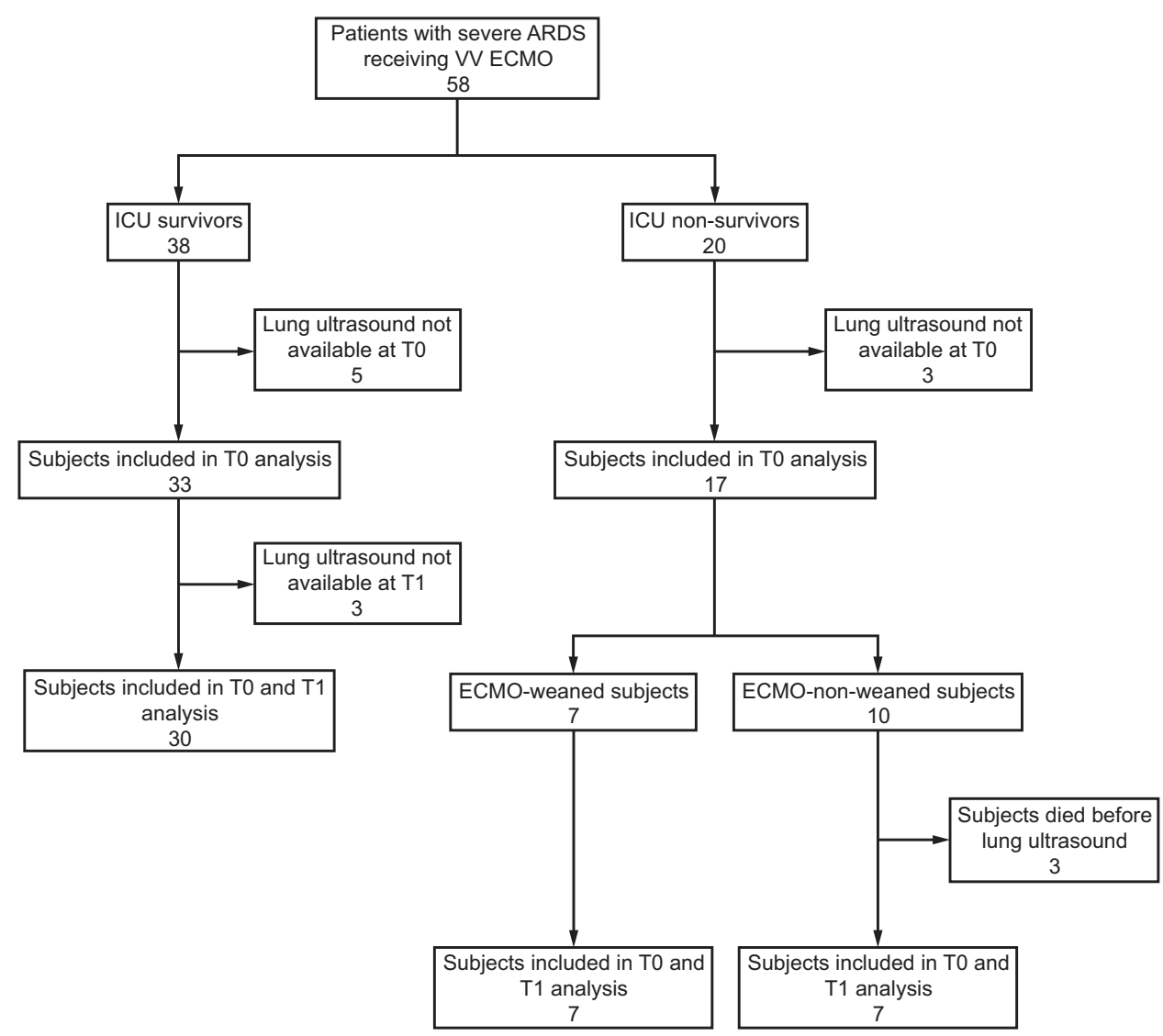

Fig. 2. Flow chart. $\mathrm{VV} E C M O=$ venovenous extracorporeal membrane oxygenation; $\mathrm{TO}=\mathrm{ECMO}$ initiation; $\mathrm{T} 1=\mathrm{ECMO}$ withdrawal.

\section{Data Collection}

The following data were collected for each subject: age, sex, body mass index, Sepsis-Related Organ Failure Assessment, Simplified Acute Physiology Score II at admission, and clinical and respiratory characteristics at the time of ECMO initiation. The duration of mechanical ventilation before ECMO placement, the duration of ECMO assistance, and treatment with neuromuscular blockade were also recorded. The total LUS, the LUS of each region of interest, and respiratory parameters were recorded at the same time points, at $\mathrm{T} 0$ and $\mathrm{T} 1$.

\section{Statistical Analysis}

The primary end point was the assessment of the LUS at initiation of ECMO for survivors and non-survivors. The secondary end points were (1) changes in the LUS from T0 to $\mathrm{T} 1,(2)$ the relationship between the changes in lung aeration and the changes in arterial oxygenation between $\mathrm{T} 0$ and $\mathrm{T} 1$, and (3) regional changes of lung aeration between $\mathrm{T} 0$ and $\mathrm{T} 1$ in the survivors. Quantitative variables between survivors and non-survivors were compared by using the Mann-Whitney U test and were expressed as median and 25-75\% interquartile range (IQR), unless otherwise specified. Categorical variables were compared by using the chi-square test or the Fisher exact test and were expressed as number and percentage. The changes of the LUS from T0 to T1 in survivors or non-survivors were compared by using the Wilcoxon signed rank tests. The relationship between changes in the LUS and $\mathrm{P}_{\mathrm{aO}} / \mathrm{F}_{\mathrm{IO}_{2}}$ between $\mathrm{T} 0$ and $\mathrm{T} 1$ was analyzed by simple linear regression.

We did an exploratory post hoc analysis in the subjects who were weaned off ECMO to compare the LUS and respiratory variables at $\mathrm{VV}$ ECMO withdrawal between survivors and non-survivors by using the Mann-Whitney $\mathrm{U}$ test. All analyses were made by using SigmaStat 3.5 (Systat Software, Point Richmond, CA) or SPSS 13.0 for Windows (SPSS, Chicago, Illinois). The statistical significance level was fixed at 0.05 .

\section{Results}

\section{Clinical Characteristics}

During the study period, a total of 58 patients with severe ARDS received VV ECMO support; 8 were excluded for lack of lung ultrasound evaluation at T0. The flow diagram is shown in Figure 2. Clinical character- 
Table 1. Clinical Characteristics of the Subjects Who Received VV ECMO

\begin{tabular}{|c|c|c|c|c|}
\hline Characteristic & $\begin{array}{l}\text { Overall } \\
(N=50)\end{array}$ & $\begin{array}{l}\text { ICU Survivors } \\
\quad(n=33)\end{array}$ & $\begin{array}{c}\text { ICU Non-Survivors } \\
\quad(n=17)\end{array}$ & $P$ \\
\hline Age, median (IQR) y & $44(25-63)$ & $37(25-51)$ & $63(41-69)$ & .006 \\
\hline Men, $n(\%)$ & $39(78)$ & $26(79)$ & $13(76)$ & $>.99$ \\
\hline SOFA admission score, median (IQR) & $12(8-14)$ & $12(9-13)$ & $13(8-15)$ & .48 \\
\hline SAPS II admission, median (IQR) & $44(37-55)$ & $42(36-56)$ & $47(42-56)$ & .27 \\
\hline BMI, median (IQR) kg/m² & $24(22-28)$ & $24(23-28)$ & $25(23-27)$ & .63 \\
\hline Cause of admission, $n(\%)$ & & & & .16 \\
\hline Surgical & $14(28)$ & $7(21.2)$ & $7(41)$ & \\
\hline Medical & $19(38)$ & $12(36.4)$ & $7(41)$ & \\
\hline Multiple trauma & $17(34)$ & $14(42.4)$ & $3(18)$ & \\
\hline Cause of ARDS, $n(\%)$ & & & & .29 \\
\hline Pulmonary & $41(82)$ & $28(85)$ & $13(76)$ & \\
\hline CAP & 9 & 4 & 5 & \\
\hline VAP & 12 & 8 & 4 & \\
\hline Aspiration & 4 & 4 & 0 & \\
\hline Lung contusion & 16 & 12 & 4 & \\
\hline Extrapulmonary & $9(18)$ & $5(15)$ & $4(24)$ & \\
\hline Shock, $n(\%)$ & $33(66)$ & $21(64)$ & $12(71)$ & .43 \\
\hline Mechanical ventilation duration before ECMO, median (IQR) d & $2.9(1.1-6.0)$ & $2.0(1.0-8.6)$ & $3.8(1.7-4.9)$ & .64 \\
\hline Pump flow (first $24 \mathrm{~h}$ ), median (IQR) L/min & $4.7(4.1-5.5)$ & $4.8(4.1-5.3)$ & $4.7(4.2-5.7)$ & .73 \\
\hline Membrane ventilation (first $24 \mathrm{~h}$ ), median (IQR) L/min & $4(4-6)$ & $4.0(4.0-6.0)$ & $5.0(3.8-5.0)$ & .77 \\
\hline $\begin{array}{l}\text { VV ECMO = venovenous extracorporeal membrane oxygenation } \\
\text { IQR = interquartile range } \\
\text { SOFA = Sequential Organ Failure Assessment } \\
\text { SAPS II = Simplified Acute Physiology II Score } \\
\text { BMI = body mass index } \\
\text { CAP = community-acquired pneumonia } \\
\text { VAP = ventilator-associated pneumonia }\end{array}$ & & & & \\
\hline
\end{tabular}

Table 2. Respiratory Parameters at VV ECMO Initiation

\begin{tabular}{|c|c|c|c|c|}
\hline Parameter & Overall $(N=50)$ & ICU Survivors $(n=33)$ & ICU Non-Survivors $(n=17)$ & $P$ \\
\hline $\mathrm{P}_{\mathrm{aO}_{2}} / \mathrm{F}_{\mathrm{IO}_{2}}, \mathrm{~mm} \mathrm{Hg}$ & $71(55-83)$ & $70(56-80)$ & $61(53-84)$ & .51 \\
\hline $\mathrm{P}_{\mathrm{aCO}_{2}}, \mathrm{~mm} \mathrm{Hg}$ & $47(40-59)$ & $48(40-59)$ & $44(39-58)$ & .64 \\
\hline $\mathrm{pH}$ & $7.29(7.17-7.37)$ & $7.32(7.06-7.38)$ & $7.26(7.21-7.33)$ & .86 \\
\hline Frequency, breaths/min & $29(25-31)$ & $28(24-31)$ & $30(26-32)$ & .61 \\
\hline $\mathrm{V}_{\mathrm{T}}, \mathrm{mL}$ & $400(338-452)$ & $430(344-470)$ & $391(335-433)$ & .41 \\
\hline $\mathrm{V}_{\mathrm{T}} / \mathrm{IBW}, \mathrm{mL} / \mathrm{kg}$ & $5.8(5.2-6.5)$ & $6.1(5.4-6.6)$ & $5.4(4.8-6.1)$ & .15 \\
\hline PEEP, $\mathrm{cm} \mathrm{H}_{2} \mathrm{O}$ & $10(8-12)$ & $12(8-13)$ & $10(8-11)$ & .18 \\
\hline $\mathrm{P}_{\text {Imax }}, \mathrm{cm} \mathrm{H}_{2} \mathrm{O}$ & $37(33-44)$ & $39(32-44)$ & $37(35-42)$ & .98 \\
\hline Inspiratory $\mathrm{P}_{\text {plat }}, \mathrm{cm} \mathrm{H}_{2} \mathrm{O}^{*}$ & $33(30-35)$ & $33(31-37)$ & $32(30-35)$ & .46 \\
\hline $\begin{array}{l}\text { Data are presented as median }(25-7 \\
* \text { For } \mathrm{P}_{\text {plat }} \text {, comparison was made } \\
\text { VV ECMO = venovenous extracorp } \\
\text { Frequency = breathing frequency } \\
\mathrm{V}_{\mathrm{T}}=\text { tidal volume } \\
\mathrm{V}_{\mathrm{T}} / \mathrm{IBW}=\text { tidal volume based on id } \\
\mathrm{P}_{\text {Imax }}=\text { maximum inspiratory press } \\
\mathrm{P}_{\text {plat }} \text { plateau pressure }\end{array}$ & $\begin{array}{l}\text { e range). } \\
\text { with available data: } 16 \text { su } \\
\text { e oxygenation }\end{array}$ & non-survivors. & & \\
\hline
\end{tabular}

istics of the subjects are summarized in Table 1. Thirtythree subjects survived to ICU discharge, with an overall ICU survival rate of $66 \%$. The non-survivors were older than the survivors. The severity scores, the causes of admission and ARDS, the presence of shock that required norepinephrine at the beginning of ECMO, and the duration of mechanical ventilation before ECMO did not significantly differ between survivors 

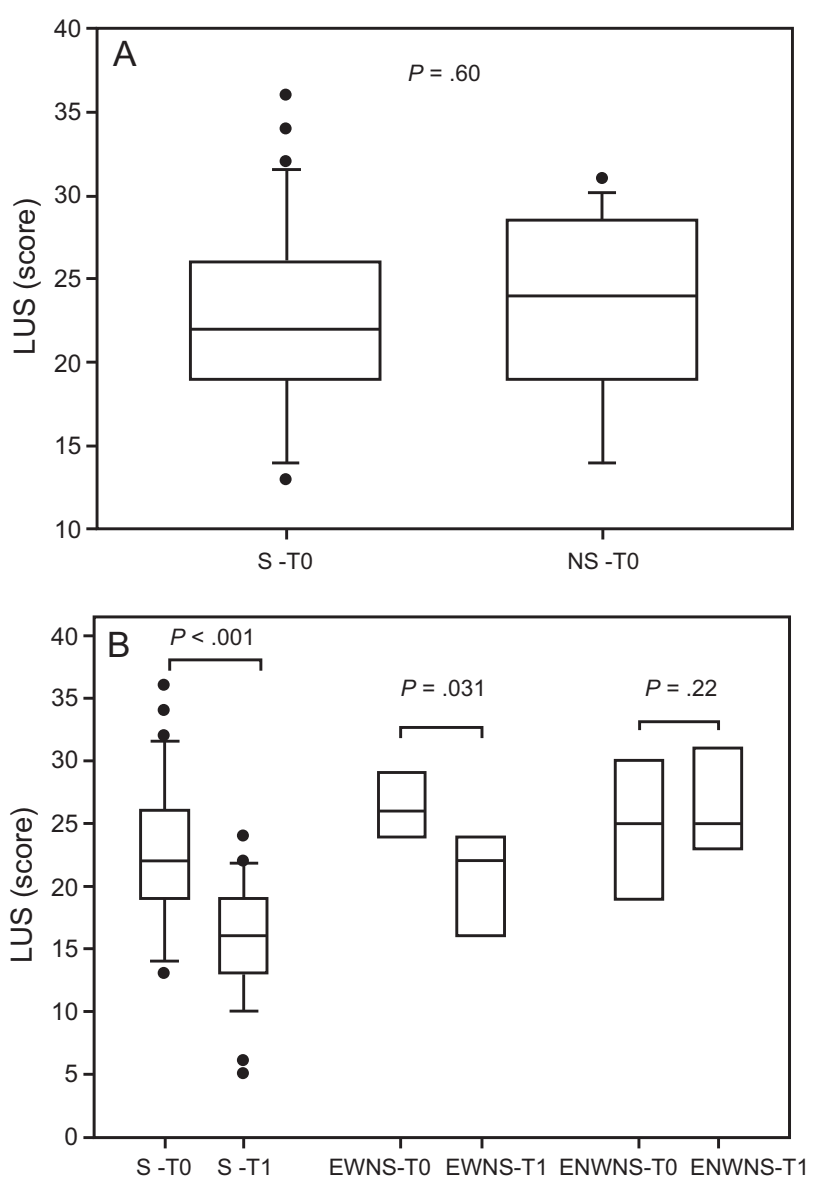

Fig. 3. A: The LUS at VV ECMO initiation (TO) in survivors and nonsurvivors. B: LUS at ECMO initiation and withdrawal in survivors and non-survivors who were weaned or not from ECMO. Data are expressed as median and $25-75 \%$ interquartile range (IQR). LUS = lung ultrasound aeration score; $\mathrm{V} \mathrm{E}$ ECMO = venovenous extracorporeal membrane oxygenation; S-TO = survivors-ECMO initiation; NS-TO = non-survivors-ECMO initiation; S-T1 = survivors-ECMO withdrawal; EWNS = ECMO-weaned non-survivors; ENWNS = ECMO non-weaned non-survivors.

and non-survivors. At T0, all the subjects were in a half-sitting position, between $30^{\circ}$ and $45^{\circ}$, and were paralyzed with atracurium. The treatment duration with neuromuscular blockade was longer in the non-survivors than in the survivors (median 7.0 [IQR, 3.0-13.8] d vs median 2.4 [IQR, 1.1-10.0] d; $P=.032$ ). At T0, the respiratory parameters and ventilator settings were not different between the survivors and the non-survivors (Table 2).

\section{Lung Aeration At ECMO Initiation and Its Changes From T0 to T1}

The LUS at ECMO initiation did not significantly differ between the survivors and non-survivors (median 22 [IQR, 19-26] vs median 24 [IQR, 19-28]; $P=.60$ ) (Fig. 3A). A significant decrease in the LUS from $\mathrm{T} 0$ to $\mathrm{T} 1$ was observed in the survivors (median 22 [IQR, 19-26] vs median 16 [13-19]; $P<.001$ ), which indicated an improvement in lung aeration at the time of ECMO withdrawal. In the non-survivors, the LUS decreased moderately in the subjects who were weaned off ECMO (median 26 [IQR, 24-29] vs median 22 [17-24]; $P=.031)$ and remained stable in those who died during the ECMO time period (median 25 [IQR, 19-29] vs median 25 [IQR, 23-31]; $P=.22$ ) (Fig. 3B). The changes in $\mathrm{P}_{\mathrm{aO}_{2}} / \mathrm{F}_{\mathrm{IO}_{2}}$ between $\mathrm{T} 0$ and $\mathrm{T} 1$ were not linearly related to the changes in the LUS (Fig. 4). The regional changes in the LUS between $\mathrm{T} 0$ and $\mathrm{T} 1$ in the survivors are shown in Figure 5. Regional lung re-aeration, as evidenced by a decrease in regional LUS occurred primarily in the anterior and upper lung areas.

\section{Post Hoc Analysis of Subjects Who Were Weaned Off ECMO}

ECMO assistance was successfully withdrawn from all survivors and 7 non-survivors. The median ECMO duration was 9 (IQR, 6-14) d. Of the 7 non-survivors, 6 were not weaned off mechanical ventilation. At ECMO withdrawal, the LUS, $\mathrm{P}_{\mathrm{aCO}_{2}}$, and maximum inspiratory pressure were significantly higher, and $\mathrm{P}_{\mathrm{aO}} / \mathrm{F}_{\mathrm{IO}_{2}}$ was significantly lower in the non-survivors than in the survivors (Table 3).

\section{Discussion}

The results of this pilot study showed that the subjects who survived to the ICU discharge and those who died in the ICU did not differ significantly for lung aeration loss at the time of ECMO initiation. In the survivors, the success of ECMO withdrawal was associated with a significant improvement in lung aeration, whereas severe loss of lung aeration persisted at ECMO withdrawal in the subjects who did not survive, although some were weaned off ECMO support. Lung re-aeration occurred primarily in anterior and upper lung areas. The changes in arterial oxygenation were not related to the changes in lung aeration.

ARDS is characterized by low respiratory compliance and reduced aerated lung volume, which results in severe hypoxemia. Patient outcome could be influenced by the severity of lung aeration loss and pulmonary compliance. ${ }^{10,22}$ It is known that the degree of hypoxemia is not always correlated with the severity of lung aeration loss due to ventilation-perfusion mismatch. ${ }^{23}$ To date, however, decision making regarding initiating and weaning off ECMO remains challenging and is largely based on the blood gas findings. ${ }^{24}$ To our knowledge, our study was the first to explore bedside assessment of lung aeration from 


\section{Ultrasound Assessment of Lung Aeration During ECMO}

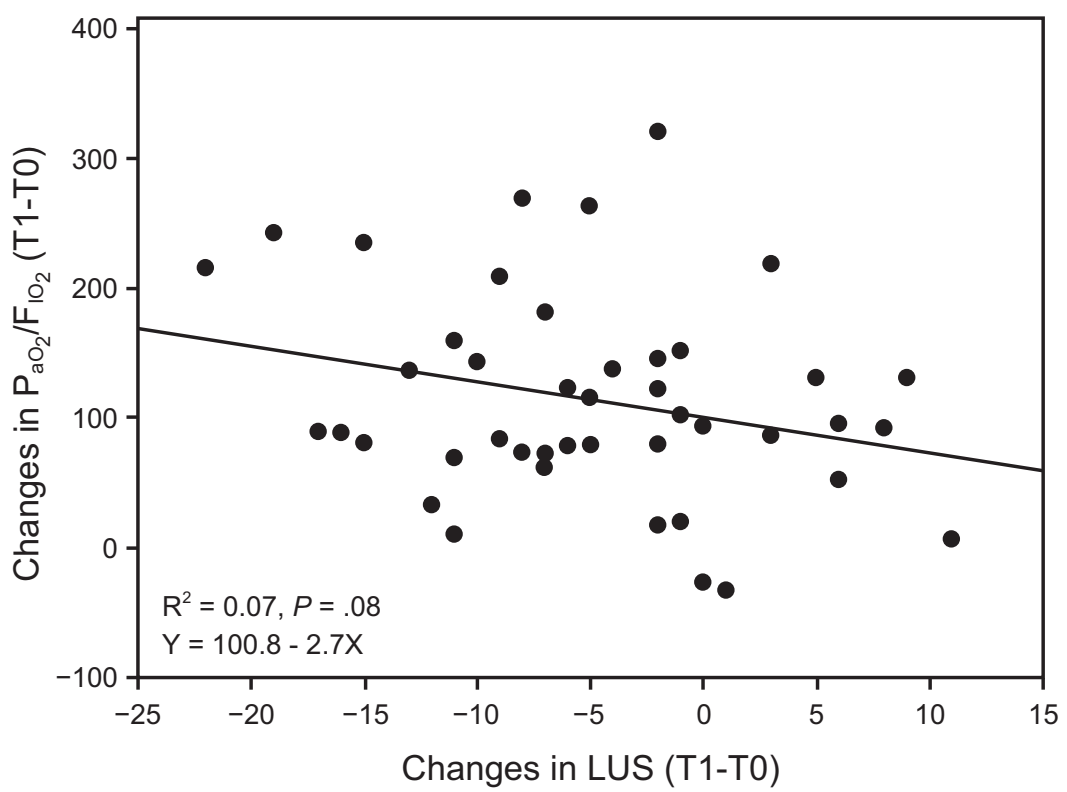

Fig. 4. Relationship between changes in the lung ultrasound aeration score and changes in $\mathrm{P}_{\mathrm{aO}_{2}} / \mathrm{F}_{1 \mathrm{O}_{2}}$ between VV ECMO initiation (TO) and withdrawal (T1). VV ECMO = venovenous extracorporeal membrane oxygenation.

the initiation to the withdrawal of ECMO and its relationship to patient outcomes by lung ultrasound.

At the ECMO onset, the loss of lung aeration and the impairment of gas exchange did not differ between the survivors and the non-survivors. In one subject who died during ECMO support, the LUS at ECMO initiation was only 14 due to the presence of diffuse interstitial edema in each lung area. These results indicated that the initial degree of lung aeration loss might not be predictive of patient outcome. At ECMO withdrawal, although it was not surprising to find a difference in severity of lung aeration between the subjects who survived ARDS and those who died during ECMO, to our knowledge, our study was the first to show these findings and to demonstrate that the LUS is a useful and valuable tool for bedside follow up on lung function in patients supported by ECMO. Of note, in the subjects who died during ECMO support, T1 corresponded to the last lung ultrasound before death; thus, this lack of improvement may suggest a poor prognosis. Interestingly, changes in the aeration score have been used to assess the effectiveness of prone positioning, and a relationship between prone-positioning response in terms of changes in lung aeration and patient prognosis was reported. ${ }^{25}$ In addition, we found that the non-survivors were older than the survivors. Some prediction models showed that older age seemed to be one of the determinants of a poor outcome. ${ }^{5,6}$

Among the 7 subjects who were initially weaned off ECMO support but who did not survive ARDS, with the exception of one subject who died suddenly from pericardial tamponade, our exploratory post hoc analysis showed a persistence of severe loss of lung aeration, with a median LUS as high as 22 during ECMO withdrawal, associated with impaired lung mechanics compared with the survivors. In addition, these subjects were not weaned off mechanical ventilation until death. Deciding when to wean off ECMO support remains highly challenging. Conventionally, ECMO is stopped according to blood gas results, ${ }^{1}$ and the recovery of lung aeration is not necessarily taken into account. Unfortunately, our results did not show any relationship between lung re-aeration and oxygenation improvement. A lack of the correlation between lung aeration changes and oxygenation responses was also found in patients with severe ARDS who had undergone prone positioning. ${ }^{25,26}$ Further prospective studies are needed to assess the clinical relevance of integrating lung aeration assessment into the ECMO weaning process and to determine whether this assessment can play a role in predicting patient outcome beyond a successful weaning off ECMO. ${ }^{13,27}$

Several limitations of the study should be discussed. First, due to the nature of the retrospective design, lung ultrasound was not systematically performed in all the subjects, which led to the exclusion of 8 subjects from the cohort for primary end point analysis. For the same reason, the LUS was not assessed at a specific time, and a range of $24 \mathrm{~h}$ around ECMO was allowed. It should be pointed out, however, in the subjects in whom LUS was assessed both before and $24 \mathrm{~h}$ after ECMO initiation, we did not find any significant difference in the LUS between these 2 time points. Second, ultrasound assessment is operator dependent. In our study, $\sim 40 \%$ of the lung ultrasound 

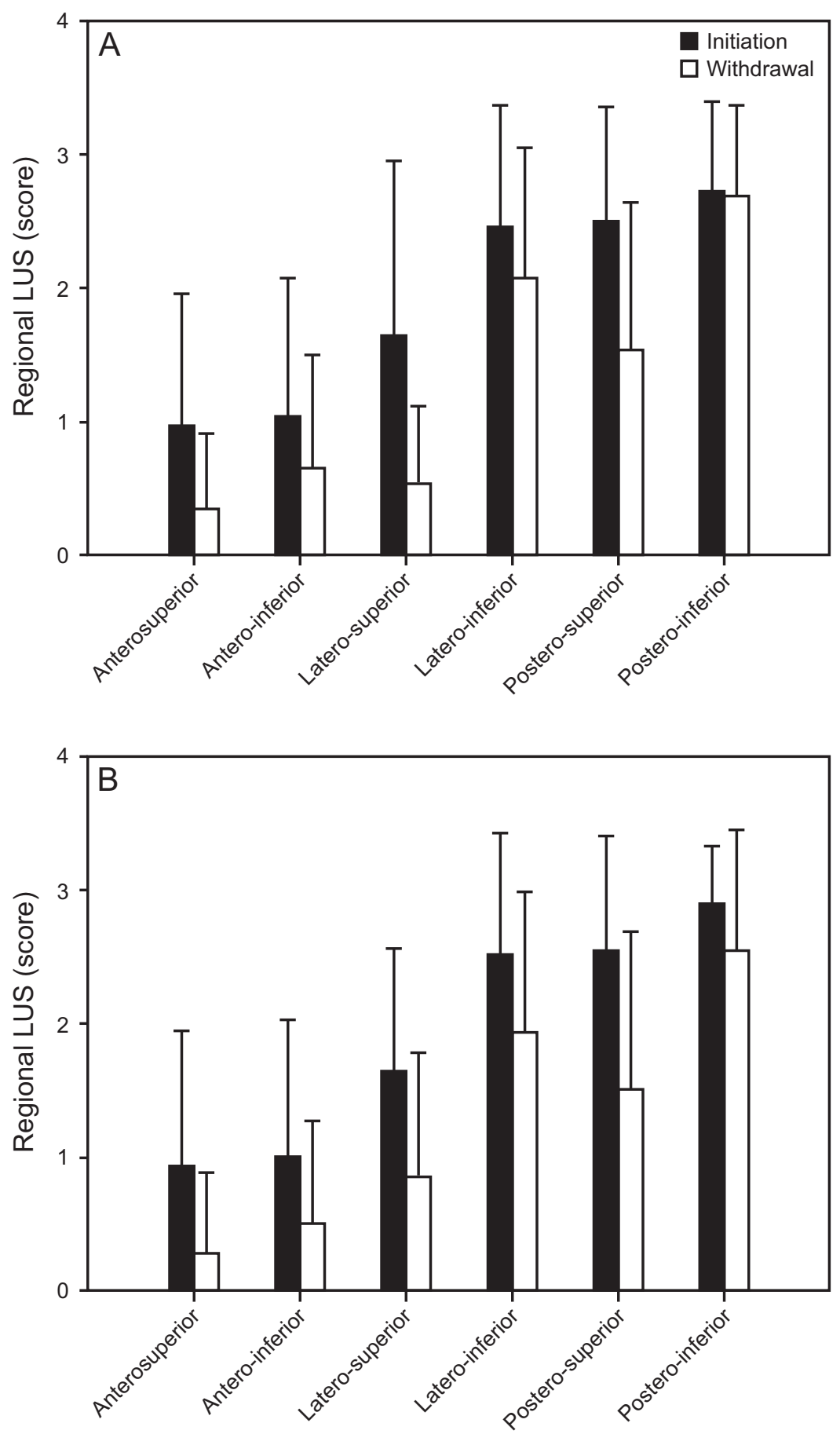

Fig. 5. Regional distribution of the lung ultrasound aeration (LUS) score assessed on 12 lung regions at the initiation and withdrawal of venovenous extracorporeal membrane oxygenation in survivors. A: Right lung. B: left lung. Data are shown as mean \pm SD.

examinations were performed by the residents who had completed lung ultrasound training, but inter-observer variability between experienced senior physicians and residents could not be assessed. It should be noted, however, a good inter-observer agreement for ultrasound assessment of lung aeration has been reported in previous studies. ${ }^{12,17}$
Furthermore, one of the limitations of lung ultrasound is a poor acoustic window in patients who are obese. ${ }^{28}$ The incidence of technically difficult echocardiography has been reported to be $45 \%$ in subjects with an increased body mass index. ${ }^{29}$ This incidence is still unknown for lung ultrasound assessment. In our study, 2 subjects had a 
Table 3. Post Hoc Analysis of Subjects Weaned Off ECMO at VV ECMO Withdrawal

\begin{tabular}{|c|c|c|c|}
\hline Parameter & $\begin{array}{l}\text { ICU Survivors } \\
\quad(n=33)\end{array}$ & $\begin{array}{l}\text { ICU Non-Survivors } \\
\quad(n=7)\end{array}$ & $P$ \\
\hline LUS & $16(13-19)$ & $22(17-24)$ & .045 \\
\hline $\mathrm{P}_{\mathrm{aO}_{2}} / \mathrm{F}_{\mathrm{IO}_{2}}, \mathrm{~mm} \mathrm{Hg}$ & $198(156-262)$ & $132(113-182)$ & .02 \\
\hline $\mathrm{P}_{\mathrm{aCO}_{2}}, \mathrm{~mm} \mathrm{Hg}$ & $38(36-42)$ & $44(43-46)$ & .03 \\
\hline $\mathrm{pH}$ & $7.43(7.39-7.47)$ & $7.36(7.31-7.42)$ & .03 \\
\hline Frequency, breaths/min & $26(22-29)$ & $26(23-29)$ & .92 \\
\hline $\mathrm{V}_{\mathrm{T}}, \mathrm{mL}$ & $393(364-442)$ & $374(352-408)$ & .49 \\
\hline $\mathrm{V}_{\mathrm{T}} / \mathrm{IBW}, \mathrm{mL} / \mathrm{kg}$ & $5.9(5.1-6.2)$ & $6.0(5.5-6.3)$ & .39 \\
\hline PEEP, $\mathrm{cm} \mathrm{H}_{2} \mathrm{O}$ & $8(7-10)$ & $8(6-10)$ & .83 \\
\hline $\mathrm{P}_{\text {Imax }}, \mathrm{cm} \mathrm{H}_{2} \mathrm{O}$ & $29(25-33)$ & $35(32-40)$ & .02 \\
\hline \multicolumn{4}{|c|}{$\begin{array}{l}\text { Data are presented as median }(25-75 \% \text { interquartile range). } \\
\text { VV ECMO = venovenous extracorporeal membrane oxygenation } \\
\text { LUS = lung ultrasound aeration score } \\
\mathrm{V}_{\mathrm{T}}=\text { tidal volume } \\
\mathrm{V}_{\mathrm{T}} / \mathrm{IBW}=\text { tidal volume based on ideal body weight } \\
\mathrm{P}_{\text {Imax }}=\text { maximum inspiratory pressure }\end{array}$} \\
\hline
\end{tabular}

body mass index $>40 \mathrm{~kg} / \mathrm{m}^{2}$ in whom lung ultrasound assessments were successfully achieved. Also, a number of factors could affect lung aeration, such as end-expiratory positive pressure, recruitment maneuvers, use of neuromuscular blockade, upper-abdominal surgery, fluid balance, prone positioning, and patient positioning during ECMO. Unfortunately, our study was unable to discern how these factors influenced the LUS and correlated with clinical outcomes. The small sample size and lower power precluded any multivariate analyses for independent risk factors. Thus, this study can be only considered as a pilot exploratory one, and the results obtained from the present study deserve further prospective investigations.

\section{Conclusions}

Transthoracic lung ultrasound provides a valuable tool for bedside assessment of lung aeration in patients with severe ARDS who are receiving VV ECMO support. Although the LUS at ECMO initiation may not be discriminating in terms of patient's severity, the assessment of its changes from ECMO onset to withdrawal may help physicians to better estimate the evolution of lung function. Further large-scale prospective studies are needed to evaluate the clinical relevance of assessing lung aeration by ultrasound in the daily management for patients with severe ARDS supported by VV ECMO.

\section{REFERENCES}

1. Combes A, Hajage D, Capellier G, Demoule A, Lavoué S, Guervilly C, et al.; EOLIA Trial Group, REVA, and ECMONet. Extracorporeal membrane oxygenation for severe acute respiratory distress syndrome. N Engl J Med 2018;378(21):1965-1975.

2. Richard C, Argaud L, Blet A, Boulain T, Contentin L, Dechartres A, et al. Extracorporeal life support for patients with acute respiratory distress syndrome: report of a Consensus Conference. Ann Intensive Care 2014;4:15.

3. Karagiannidis C, Brodie D, Strassmann S, Stoelben E, Philipp A, Bein T, et al. Extracorporeal membrane oxygenation: evolving epidemiology and mortality. Intensive Care Med 2016;42(5):889-896.

4. Peek GJ, Mugford M, Tiruvoipati R, Wilson A, Allen E, Thalanany $\mathrm{MM}$, et al.; CESAR trial collaboration. Efficacy and economic assessment of conventional ventilatory support versus extracorporeal membrane oxygenation for severe adult respiratory failure (CESAR): a multicentre randomised controlled trial. Lancet 2009;374(9698):1351-1363.

5. Baek MS, Chung CR, Kim HJ, Cho WH, Cho YJ, Park S, et al. Age is major factor for predicting survival in patients with acute respiratory failure on extracorporeal membrane oxygenation: a Korean multicenter study. J Thorac Dis 2018;10(3):1406-1417.

6. Rozencwajg S, Pilcher D, Combes A, Schmidt M. Outcomes and survival prediction models for severe adult acute respiratory distress syndrome treated with extracorporeal membrane oxygenation. Crit Care 2016;20(1):392.

7. Schmidt M, Bailey M, Sheldrake J, Hodgson C, Aubron C, Rycus $\mathrm{PT}$, et al. Predicting survival after extracorporeal membrane oxygenation for severe acute respiratory failure. The Respiratory Extracorporeal Membrane Oxygenation Survival Prediction (RESP) score. Am J Respir Crit Care Med 2014;189(11):1374-1382.

8. Serpa Neto A, Schmidt M, Azevedo LC, Bein T, Brochard L, Beutel G, et al.; ReVA Research Network and the PROVE Network Investigators. Associations between ventilator settings during extracorporeal membrane oxygenation for refractory hypoxemia and outcome in patients with acute respiratory distress syndrome: a pooled individual patient data analysis: mechanical ventilation during ECMO. Intensive Care Med 2016;42(11):1672-1684.

9. Mrozek S, Jabaudon M, Jaber S, Paugam-Burtz C, Lefrant JY, Rouby JJ, et al.; Azurea network. Elevated plasma levels of sRAGE are associated with nonfocal CT-based lung imaging in patients with ARDS: a prospective multicenter study. Chest 2016;150(5):998-1007.

10. Rouby JJ, Puybasset L, Cluzel P, Richecoeur J, Lu Q, Grenier P. Regional distribution of gas and tissue in acute respiratory distress syndrome. II. Physiological correlations and definition of an ARDS Severity Score. Intensive Care Med 2000;26(8):1046-1056.

11. Bouhemad B, Brisson H, Le-Guen M, Arbelot C, Lu Q, Rouby JJ. Bedside ultrasound assessment of positive end-expiratory pressure-in- 


\section{Ultrasound Assessment of Lung Aeration During ECMO}

duced lung recruitment. Am J Respir Crit Care Med 2011;183(3):341347.

12. Bouhemad B, Liu ZH, Arbelot C, Zhang M, Ferarri F, Le-Guen M, et al. Ultrasound assessment of antibiotic-induced pulmonary reaeration in ventilator-associated pneumonia. Crit Care Med 2010;38(1):84-92.

13. Soummer A, Perbet S, Brisson H, Arbelot C, Constantin JM, Lu Q, et al.; Lung Ultrasound Study Group. Ultrasound assessment of lung aeration loss during a successful weaning trial predicts postextubation distress*. Crit Care Med 2012;40(7):2064-2072.

14. Mongodi S, Pozzi M, Orlando A, Bouhemad B, Stella A, Tavazzi G, et al. Lung ultrasound for daily monitoring of ARDS patients on extracorporeal membrane oxygenation: preliminary experience. Intensive Care Med 2018;44(1):123-124.

15. ARDS Definition Task Force, Ranieri VM, Rubenfeld GD, Thompson BT, Ferguson ND, Caldwell E, et al. Acute respiratory distress syndrome: the Berlin Definition. JAMA 2012;307(23):2526-2533.

16. Neri L, Storti E, Lichtenstein D. Toward an ultrasound curriculum for critical care medicine. Crit Care Med 2007;35(5 Suppl):S290S304.

17. Rouby JJ, Arbelot C, Gao Y, Zhang M, Lv J, An Y, et al. Training for lung ultrasound score measurement in critically ill patients. Am J Respir Crit Care Med 2018;198(3):398-401.

18. Lichtenstein D, Mezière G, Seitz J. The dynamic air bronchogram. A lung ultrasound sign of alveolar consolidation ruling out atelectasis. Chest 2009;135(6):1421-1425.

19. Arbelot C, Ferrari F, Bouhemad B, Rouby JJ. Lung ultrasound in acute respiratory distress syndrome and acute lung injury. Curr Opin Crit Care 2008;14(1):70-74.

20. Caltabeloti F, Monsel A, Arbelot C, Brisson H, Lu Q, Gu WJ, et al. Early fluid loading in acute respiratory distress syndrome with septic shock deteriorates lung aeration without impairing arterial oxygenation: a lung ultrasound observational study. Crit Care 2014;18(3): R91.
21. Combes A, Bacchetta M, Brodie D, Müller T, Pellegrino V. Extracorporeal membrane oxygenation for respiratory failure in adults. Curr Opin Crit Care 2012;18(1):99-104.

22. Jabaudon M, Blondonnet R, Pereira B, Cartin-Ceba R, Lichtenstern $\mathrm{C}$, Mauri T, et al. Plasma sRAGE is independently associated with increased mortality in ARDS: a meta-analysis of individual patient data. Intensive Care Med 2018;44(9):1388-1399.

23. Putensen C, Mutz NJ, Putensen-Himmer G, Zinserling J. Spontaneous breathing during ventilatory support improves ventilation-perfusion distributions in patients with acute respiratory distress syndrome. Am J Respir Crit Care Med 1999;159(4 Pt 1):1241-1248.

24. Mols G, Loop T, Geiger K, Farthmann E, Benzing A. Extracorporeal membrane oxygenation: a ten-year experience. Am J Surg 2000; 180(2):144-154.

25. Wang XT, Ding X, Zhang HM, Chen H, Su LX, Liu DW; Chinese Critical Ultrasound Study Group (CCUSG). Lung ultrasound can be used to predict the potential of prone positioning and assess prognosis in patients with acute respiratory distress syndrome. Crit Care 2016;20(1):385.

26. Haddam M, Zieleskiewicz L, Perbet S, Baldovini A, Guervilly C, Arbelot C, et al.; CAR'Echo Collaborative Network, AzuRea Collaborative Network. Lung ultrasonography for assessment of oxygenation response to prone position ventilation in ARDS. Intensive Care Med 2016;42(10):1546-1556.

27. Schmidt M, Stewart C, Bailey M, Nieszkowska A, Kelly J, Murphy L, et al. Mechanical ventilation management during extracorporeal membrane oxygenation for acute respiratory distress syndrome: a retrospective international multicenter study. Crit Care Med 2015;43(3):654-664.

28. Koenig SJ, Narasimhan M, Mayo PH. Thoracic ultrasonography for the pulmonary specialist. Chest 2011;140(5):1332-1341.

29. Supariwala A, Makani H, Kahan J, Pierce M, Bajwa F, Dukkipati SS, et al. Feasibility and prognostic value of stress echocardiography in obese, morbidly obese, and super obese patients referred for bariatric surgery. Echocardiography 2014;31(7):879-885.

This article is approved for Continuing Respiratory Care Education credit. For information and to obtain your CRCE

(free to AARC members) visit www.rcjournal.com

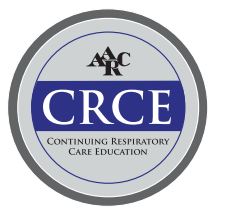

